\title{
Paisaje lingüístico en Benín: un recurso didáctico y motivador para el aula de lenguas extranjeras
}

\author{
Laurent-Fidèle Sossouvi y Mei-Chih Lin (Cotonou)
}

\begin{abstract}
Multilingualism and multiculturalism have been and are constitutive aspects of African societies. This pioneer study analyzes the linguistic landscapes of Cotonou and Abomey-Calavi (Benin), two contiguous cities; in order to verify the status and the vitality of the languages used and spoken in the country as well as seeing if it is possible to exploit didactically this written modality. For this purpose, a quantitative analysis of written language productions in public space was carried out. The results reveal the languages used in the urban scene of both cities as well as the linguistic contact in their diverse aspects. They also indicate that the linguistic landscape doesn't take into account the local multilingual practices in all their complexity. It appears as a context of learning which can be used as a didactic resource in the teaching of foreign languages.
\end{abstract}

\section{$1 \quad$ Introducción}

La ciudad constituye un lugar de contacto entre lenguas (cf. Backhaus 2007: 1), nos susurra y lo hace con su inagotable riqueza lingüística, voces, códigos, soportes complejos que determinan su identidad, la de sus residentes. Estos últimos están rodeados de paisaje lingüístico (PL) sin ser plenamente consciente de ello. Para ajustar a la demanda de la globalización avanzada, de movilidad de recursos humanos y materiales, el proceso de enseñanza-aprendizaje se centra cada vez más en las necesidades e intereses de los aprendientes, así como en la interculturalidad. Como es sabido, desde la primera infancia, los benineses se enfrentan a una educación multilingüe, consecuencia de la convivencia de varias lenguas de estatus distinto: las lenguas autóctonas o nacionales, la lengua francesa, la única lengua oficial, las lenguas extranjeras áulicas o no, las lenguas esotéricas o secretas y las lenguas alóctonas (cf. Sossouvi 2014, 2016, 2017). Todas estas lenguas y variedades cohabitan de modo pacífico, pese a que la lengua francesa es la más visible, la más legible. Por ello, creemos que los discentes de lenguas extranjeras (LEs) pueden recoger conocimiento de su entorno, en este caso a partir del paisaje lingüístico.

Este concepto hace referencia a los textos escritos en las vías públicas, el lenguaje visible en el entorno público y es un símbolo de cohabitación entre dos o más culturas. Es un acto comunicativo e incluso un importante medio de representación afectiva. Las investigaciones sobre paisajes lingüísticos son relativamente recientes y aunque la bibliografía sobre estos cada vez es más abundante, aún no existe una teoría consensuada. No obstante, hoy por hoy 
progresivamente, el PL se ha ido incorporando en los diseños curriculares de la enseñanza formal (cf. Cenoz/Gorter 2008, Sabatier/Moore/Dagenais 2013). Es un área que cobra cada vez más importancia en la lingüística aplicada. Así, en este trabajo, queremos utilizar estas muestras escritas como indicio orientativo para determinar en qué medida están representadas las lenguas presentes en los espacios públicos de Cotonú y Abomey-Calavi, dos ciudades contiguas; qué posición ocupan estas lenguas y comprobar si pueden servir de apoyo o herramienta didáctica. Más concretamente, estas páginas pretenden describir el uso de textos en sus espacios públicos, mostrar analíticamente su multilingüismo y ver si se puede explotar didácticamente esta modalidad escrita.

El paisaje lingüístico de Cotonú y Abomey-Calavi parece particularmente interesante porque la primera ciudad es la capital económica de Benín y la siguiente, el segundo municipio más poblado del país. Ambas urbes han experimentado en los últimos años importantes cambios socioeconómicos y demográficos, debidos a la movilidad de recursos humanos, a las actividades internacionales, etc. Por lo tanto, representan una región mixta donde los locutores de diversas lenguas comparten espacio y su PL deja ver un escenario expresivo elaborado.

Cabe señalar que, por razones de espacio, la descripción de esta investigación va a ser más textual que iconográfica y se confinará a presentaciones generales. De la misma manera, descartamos el paisaje auditivo de ambas ciudades. Comenzaremos exponiendo el marco teórico, aproximando al concepto de paisaje lingüístico y a la revisión de algunas investigaciones realizadas acerca del tema. A continuación, se presentará el estudio empírico que nos ayuda a conocer el contexto de la investigación, los instrumentos y procedimientos utilizados. Después se ofrecerán los hallazgos significativos obtenidos y, por último, se intentará discutir los resultados más importantes.

\section{$2 \quad$ Marco teórico}

\section{1 ¿Qué se entiende por paisaje lingüístico?}

El concepto de paisaje lingüístico se ha desarrollado en los últimos años en el enfoque semiótico y sociolingüístico independiente (cf. Boschung 2016), en las teorías de los signos formuladas por los estructuralistas y un desarrollo dentro de la lingüística aplicada (cf. Pons Rodríguez 2012). Consiste en describir la utilización de cualquier tipo de manifestaciones de textos o lengua escrita en el espacio público urbano. Concretamente, el PL designa toda lengua de los anuncios públicos, carteles viarios, escaparates, vallas publicitarias, letreros públicos y comerciales, rótulos de calles y carreteras, señalización de edificios públicos, $u$ otras inscripciones que están presentes en los espacios públicos. Por lo tanto, engloba las lenguas visibles en la esfera pública, que nos informan incesante e inconscientemente sobre las características culturales de nuestro entorno. En otras palabras, es un tipo de arte urbano que busca dejar un mensaje comercial, político, social, económico, demográfico o cultural en un espacio público.

Según Landry/Bourhis (1997) y Bourhis/Landry (2002), el PL desempeña dos papeles sociales, a saber: informativo o denotativo y simbólico o connotativo. El primero tiene que ver con todo lo que el paisaje lingüístico puede informar sobre los moradores y los usuarios de este sitio (cf. Landry/Bourhis 1997) e informa al público. Por ejemplo, la señalización geográfica indica el 
conjunto del territorio de una comunidad y los carteles públicos, privados o comerciales venden un servicio o informan al público. En cuanto a la función simbólica, se refiere a la potencia y al estatus de la lengua utilizada, es decir, su prestigio y vitalidad etnolingüística. Muy a menudo el PL refleja el valor y el estatus asociado a las lenguas coexistentes en el país. A modo de ejemplo, si el español no se usa en el paisaje lingüístico de una comunidad hispanófona puede deducirse que el estatus, el valor o el poder otorgado a la lengua castellana en esta comunidad es débil. Por consiguiente, esta modalidad escrita constituye un indicador de la vitalidad lingüística e incluso cultural en una sociedad multilingüe. Puede evidenciar la preponderancia y el estatus de las distintas lenguas que coexisten en esta comunidad, así como la globalización, esto es, la presencia de lenguas dominantes.

La existencia del PL en una ciudad es un buen barómetro de las transformaciones sociolingüísticas, política y planificación lingüística, y de mezcla cultural. Puede evidenciar la penetración y el establecimiento de los inmigrantes en el país o huellas del turismo (cf. Zas Varela/Prego Vázquez 2018). Además, el PL puede servir para examinar los micromachismos, los microrracismos y las microviolencias lingüísticas presentes en la sociedad (cf. Prego Vázquez/Zas Varela 2018: 278).

De ahí el PL aparece como una buena brújula para estudiar la política lingüística o la legislación lingüística de un país. Efectivamente, en palabras de Cenoz/Gorter (2006: 68), "The study of the linguistic landscape can also be interesting because it can provide information on the differences between the official language policy that can be reflected in top-down signs such as street names or names of official buildings and the impact of that policy on individuals as reflected in bottom-up signs such as shops names or street posters". En resumidas cuentas, un estudio sobre el PL mide la importancia relativa de cada lengua presente en el espacio público y no solo es una indicación de su estatuto, sino también de su dinamismo. Su estudio nos permite entender mejor los fenómenos de plurilingüismo o multilingüismo y puede servir como recurso fiable para el aprendizaje de lenguas extranjeras (LEs) cada vez más autónomo, complejo y competitivo.

\subsection{Antecedentes}

Desde los años noventa se ha venido prestando una creciente atención a los carteles, los rótulos de las tiendas y negocios privados, los textos escritos en las vías públicas, etc., en lo que se ha denominado "paisaje lingüístico". Su estudio se ha desarrollado en el siglo XX, ya que han surgido paisajes lingüísticos que forman parte del panorama formado por el ser humano e incluso queda una particularidad del mundo urbano (cf. Boschung 2016: 161). El fenómeno de la migración humana contribuyó a enmarañar estos paisajes. Los trabajos sobre el PL se centran, por lo tanto, en valorar la trascendencia de cada lengua en los textos del espacio urbano. La toma en consideración de estas lenguas en el PL es un indicio no solo de su estatus, sino también de su vitalidad (cf. Bourhis/Landry 2002, Sabatier/Moore/Dagenais 2013). No obstante, cabe señalar que la aparición de una lengua en el PL de una zona no basta para justificar su vitalidad en la misma (cf. Barni/Bagna 2015, Blackwood 2015).

El comienzo de los estudios sobre el PL se produjo con Landry/Bourhis (1997) (cf. Sabatier/Moore/Dagenais 2013, Cormier 2015). Estos precursores sentaron las bases teóricas de esta modalidad escrita a partir de datos empíricos. Estudiaron el PL como una manifestación 
del estatus de la lengua de la minoría, pues se preocuparon por los factores de vitalidad etnolingüística. Con todo, cabe mencionar que también la literatura científica ha identificado otros trabajos fundacionales del campo, como los de Masai (1972) por estudiar la presencia del inglés en Tokio; de Rosenbaum et al. (1977) y Spolsky/Cooper (1991), por analizar el PL de Jerusalén; y por fin el de Tulp (1978) por examinar la posición de la lengua de Molière en Bruselas.

Cenoz/Gorter (2006) cotejaron el PL vasco con el holandés e investigaron la plaza que ocupan el euskara en España y el frisio en los Países Bajos, dos lenguas minoritarias, en el PL de sus distintas regiones. Llegaron a la conclusión de que el PL reflejaba esencialmente el contexto sociolingüístico del grupo minoritario. Además, mostraron que estas lenguas estaban infrarrepresentadas en las regiones donde se hablan.

En cuanto a Sáez Rivera/Castillo Lluch (2012) hicieron un trabajo pionero sobre las lenguas presentes en el PL de Madrid. Su análisis se basa en un corpus de fotos para describir la multiculturalidad y el multilingüismo que tienen lugar en los espacios públicos madrileños.

Sabatier/Moore/Dagenais (2013) apuntaron que, en el ámbito escolar, el trabajo sobre el PL sirve para enseñar a los aprendientes a observar el paisaje urbano plurilingüe de su comunidad, preguntarse sobre la existencia del plurilingüismo y para hacer y fomentar un discurso sobre la valorización y la desvalorización de las lenguas en su comunidad en situaciones de minorización o discriminación lingüística.

Cormier (2015) analiza el PL de Saint-Boniface, Manitoba, una comunidad francófona minoritaria, cuyos resultados muestran que la lengua francesa tiene un estatus de lengua minoritaria y que todavía existen tensas relaciones entre francófonos y anglófonos de la ciudad de Winnipeg.

Por último, Pujol Berché/Rámila Díaz (2018) estudiaron algunos estereotipos sobre España en el PL de comercios de París, con el fin de analizar su instrumentalización. Sus resultados indicaron que el uso de la lengua cervantina estaba equilibrado por el de la lengua francesa, rectificando la fuerza de política lingüística gala. El análisis de los estereotipos señala que los símbolos y las imágenes escogidos evidencian un universo folklórico e invariable.

Dicho brevemente, los estudios sobre el PL son recientes, no tienen una larga tradición; sin embargo, son un campo que sigue y seguirá desarrollándose con una variedad de enfoques y temas. Para nosotros, el PL puede utilizarse también como instrumento pedagógico para enseñar lenguas no maternas (cf. Rowland 2013, Malinowski 2015, entre otros), para la educación multicultural (cf. Hancock 2012) e incluso la posibilidad de actuar sobre la consolidación de las competencias lingüística, comunicativa y pragmática del aprendiente de lenguas extranjeras.

\section{$3 \quad$ El estudio empírico}

\subsection{El contexto}

Cotonú y Abomey-Calavi se encuentran en una zona lingüísticamente mixta, multilingüe y son las dos urbes más cosmopolitas y globales de Benín. La elección de estas dos ciudades contiguas parece imponerse, ya que en estas varias culturas y lenguas se encuentran y cohabitan. Además, 
las calles escogidas son muy concurridas y se pueden sacar fotos de numerosos letreros, carteles, vallas publicitarias, tablones de anuncios, tanto públicos, privados como comerciales. Ambas ciudades se componen de un número bastante grande de establecimientos privados y públicos, así como de tiendas y servicios prestados por todas las comunidades lingüísticas nacionales e internacionales. En las últimas décadas, han conocido unas dinámicas transformaciones demográficas, sociolingüísticas, económicas que se han expresado evidentemente en su PL multilingüe. Constituyen un ejemplo de procesos de elitización residencial en el país. Cabe señalar que albergan asimismo las mejores escuelas y universidades del país.

Cotonú es la capital económica, la ciudad más importante y conocida del país. Por su situación geográfica e infraestructuras es un centro económico y financiero del país e incluso para el comercio de África occidental. Es la sede del gobierno, de embajadas, consulados, grandes empresas y bancos del país. En cuanto a Abomey-Calavi, se encuentra al norte de Cotonú, a tan solo unos metros de esta urbe. Es el segundo municipio más poblado de Benín (INSAE 2015: 14-15), además de ser una ciudad dormitorio debido a su fuerte crecimiento demográfico, el éxodo de algunos habitantes de Cotonú, su relativa tranquilidad y el precio más asequible de las parcelas, viviendas, del alquiler de casas y pisos.

Nuestro estudio cubre el paisaje lingüístico de la carretera que empieza desde la glorieta que va a casa del expresidente Mathieu Kérékou, en el barrio Zopah (Abomey-Calavi), pasando por la iglesia Bon Pasteur, el Complejo Hospitalario Universitario de Cotonú, el Centro Cultural Chino, la iglesia Notre Dame hasta el puente de peaje, con sus calles adyacentes, o sea una distancia de unos $37 \mathrm{~km}$.

\subsection{Instrumentos de recogida de datos}

Hemos utilizado la observación y el análisis estadístico para recoger los datos de esta investigación. La observación nos ha permitido obtener informaciones de primera mano de parte del paisaje lingüístico de Cotonú y Abomey-Calavi. Los tres diarios de campo utilizados eran cuadernos de tapas duras en los que registrábamos algunos signos verbales encontrados a lo largo de nuestro itinerario, anotamos elementos importantes sobre la investigación. Hemos recurrido a otros materiales como dos cámaras de fotos, una videocámara, cuatro móviles inteligentes y tres motos. Las cámaras sirvieron para sacar las fotos de algunos textos del PL con el fin de constituir un corpus de fotografías. La videocámara nos sirvió para grabar, transmitir el PL y tener videos digitales para un análisis posterior. Utilizamos los móviles inteligentes para capturar imágenes, para comunicarnos por llamadas o por videollamadas. Apostamos por las motos para movernos por la ciudad, porque son un elemento clave en la movilidad urbana eficiente. Permiten usar racionalmente el espacio, contaminan menos, facilitan los desplazamientos y se pueden aparcarlas más fácilmente.

\subsection{Procedimientos}

La primera fase de la encuesta tuvo lugar el martes, $1^{\circ}$ de marzo de 2016 y la segunda, casi tres meses más tarde, el lunes, 30 de mayo de 2016. La primera etapa tuvo lugar en plena campaña de las elecciones presidenciales beninesas de 2016 y la segunda después de la toma de posesión del actual presidente Patrice Talon. Las dos fases empezaron a las nueve y media de la mañana 
con el fin de asegurar que las tiendas estaban abiertas. De este modo, se podría fotografiar y contabilizar las manifestaciones escritas. Para identificar los textos del PL nos basamos en las definiciones dadas por Backhaus (2006: 55), a saber: "A sign was considered to be any piece of written text within a spatially definable frame" y por Sabatier/Moore/Dagenais (2013: 141), "L'emphase sur l'information écrite sous toutes ses formes (mots, graphes, images, autres éléments visuels, etc.) et présente au sein d'un périmètre" ('El énfasis en la información escrita en todas sus formas (palabras, grafos, imágenes, otros elementos visuales, etc.) y presente dentro de un área limitada'1). Es un estudio del PL en el sentido amplio, porque no solo analizamos los elementos escritos e inertes, sino también consideramos las inscripciones y escritos sobre la ropa de los viandantes, los comerciantes, clientes sentados, etc. Al igual que otros estudios sobre el PL como el de Regueira/López Docampo/Wellings (2013), tuvimos en cuenta los nombres propios, nombres de marcas o de tiendas, ya que los emisores de estos textos podrían elegir otro nombre para sus negocios, pues, lo hicieron conscientemente, además son un componente muy habitual en el PL de Benín. Solo excluimos los textos interiores y los que no estaban expuestos a la calle.

El corpus de textos obtenidos se ha distribuido tomando en consideración las lenguas utilizadas en los espacios públicos, así como la relación cuantitativa y cualitativa entre las lenguas. Es importante señalar que la clasificación de los elementos constituyentes del PL no es siempre evidente, ya que registramos palabras yuxtapuestas, abreviaturas y nos topamos con dificultades para distinguir entre préstamos y las alternancias de códigos. Pero nuestro conocimiento de las distintas lenguas presentes en el país y las preguntas que hicimos a algunos dueños de tiendas facilitaron la comprensión del contenido de los mensajes.

Para la realización de la primera fase de la investigación, nos ayudaron tres amigos ${ }^{2}$, profesores de ELE a tiempo parcial en distintos Institutos de Educación Secundaria de ambas ciudades, cuya edad media es de 23 años. Tienen el título de Maestría en estudios hispánicos por la universidad de Abomey-Calavi y hablan francés, varias lenguas locales, varias lenguas extranjeras como el inglés, el español y tienen conocimientos del portugués y chino mandarín. $\mathrm{Su}$ papel era contabilizar y fotografiar los elementos del paisaje lingüístico. Para hacer la recogida, nos dividimos en dos grupos totalmente aleatorios: el grupo 1 constaba de los tres ayudantes, mientras que el grupo 2 comprendía los dos autores de este trabajo. A la ida el grupo 1 se ocupó del lado derecho de la calle y el grupo 2 se encargó del lado izquierdo, mientras que a la vuelta intercambiaron de lado, solo para comprobar el cálculo del otro. A las seis de la tarde, nos juntamos para hacer balance de nuestra encuesta.

La segunda etapa era un intervalo de control y solo intervinieron los dos autores. El objetivo consiste en revisitar, contabilizar de nuevo los textos del PL, comprobar si la distribución de las lenguas implicadas era estable o cambiante e identificar cambios en la fase anterior. Hemos constituido un corpus fotográfico únicamente con textos que contenían las distintas lenguas del PL. El corpus de fotos se creó en varios momentos (las fechas señaladas anteriormente) y seguimos ampliándolo a nuevos estudios venideros. Es importante señalar que este primer trabajo solo ha optado por la representatividad de las lenguas en las dos ciudades.

\footnotetext{
${ }^{1}$ La traducción es nuestra.

2 Es el lugar de agradecerlos encarecidamente la ayuda.
} 


\section{$4 \quad$ Hallazgos}

La investigación que presentamos se ciñe a los elementos relacionados con el paisaje lingüístico de Cotonú y Abomey-Calavi. A continuación, se exponen los hallazgos de particular relevancia del estudio. Hemos podido observar cerca de 24 lenguas en los espacios públicos y recoger un corpus de unos 3.109 textos mensajes de PL en el espacio estudiado: 2.364 textos monolingües (76,04\%), 674 textos bilingües $(21,68 \%), 54$ textos trilingües $(1,74 \%), 14$ textos cuatrilingües $(0,45 \%)$ y tres textos quintilingües de PL $(0,09 \%)$.

\section{- Textos monolingües}

Los elementos monolingües documentados (por ejemplo, la foto 11) alcanzaron 2.364 y se refieren al francés $(n=1975$ casos), al inglés $(n=225$ casos), a la lengua china $(n=99$ casos), a la lengua cervantina ( $n=61$ casos $)$ y otras lenguas $(n=$ cuatro casos $)$.

\section{- Textos bilingües}

Los textos bilingües documentados (por ejemplo, las fotos 2, 3, 6, 8, 10) alcanzaron 674 y tienen que ver con el uso del francés junto con el inglés ( $n=356$ casos), el PL bilingüe francés-fongbe ( $n=110$ casos), el PL bilingüe chino-francés ( $n=65$ casos), el PL bilingüe español-francés ( $n$ $=41$ casos), el PL bilingüe inglés-yoruba ( $n=31$ casos), el PL bilingüe inglés-español ( $n=25$ casos), el PL bilingüe chino-inglés ( $\mathrm{n}=24$ casos), el PL bilingüe inglés-alemán $(\mathrm{n}=20$ casos), el PL bilingüe francés-árabe ( $\mathrm{n}=$ un caso) y el PL bilingüe latín-francés $(\mathrm{n}=$ un caso).

\section{- Textos trilingües}

Los elementos trilingües registrados (por ejemplo, las fotos 7, 12) alcanzaron 54 y se refieren al PL trilingüe inglés-francés-yoruba ( $\mathrm{n}=15$ casos), al francés-español-inglés ( $\mathrm{n}=12$ casos), al chino-inglés-francés ( $\mathrm{n}=11$ casos), al inglés-gungbe-francés ( $\mathrm{n}=10$ casos), al francésfongbe-inglés ( $\mathrm{n}=$ cuatro casos $)$ y al inglés-francés-alemán $(\mathrm{n}=$ dos casos $)$.

\section{- Textos cuatrilingües}

Documentamos 14 textos cuatrilingües (ver por ejemplo, la foto 1) en el PL investigado y conciernen al chino-inglés-francés-español ( $\mathrm{n}=11$ casos) y al francés-gungbe-inglés-fongbe ( $\mathrm{n}$ $=$ tres casos).

\section{- Textos quintilingües}

De todos los ejemplos que componen el corpus solo había tres carteles con esta característica; en uno está presente el inglés junto con el francés, el castellano, el chino y el portugués (n = dos casos) y en el otro el chino está junto al inglés, el francés, el español y el árabe ( $\mathrm{n}=$ un caso). La Tabla 1 recapitula las lenguas que están representadas en el PL de las calles estudiadas. 


\begin{tabular}{|c|c|c|}
\hline 1. Textos monolingües & 2364 & $100 \%$ \\
\hline Francés & 1975 & $83,54 \%$ \\
\hline Inglés & 225 & $9,52 \%$ \\
\hline Chino & 99 & $4,19 \%$ \\
\hline Español & 61 & $2,58 \%$ \\
\hline Otras lenguas & 04 & $0,17 \%$ \\
\hline 2. Textos bilingües & 674 & $100 \%$ \\
\hline Francés-inglés & 356 & $52,82 \%$ \\
\hline Francés- fongbe & 110 & $16,32 \%$ \\
\hline Chino-francés & 65 & $9,64 \%$ \\
\hline Español-francés & 41 & $6,08 \%$ \\
\hline Inglés-yoruba & 31 & $4,60 \%$ \\
\hline Inglés-español & 25 & $3,71 \%$ \\
\hline Chino-inglés & 24 & $3,56 \%$ \\
\hline Inglés-alemán & 20 & $2,97 \%$ \\
\hline Francés-árabe & 01 & $0,15 \%$ \\
\hline Latín-francés & 01 & $0,15 \%$ \\
\hline 3. Textos trilingües & 54 & $100 \%$ \\
\hline Inglés-francés-yoruba & 15 & $27,78 \%$ \\
\hline Francés-español-inglés & 12 & $22,22 \%$ \\
\hline Chino-inglés-francés & 11 & $20,37 \%$ \\
\hline Inglés-gungbe-francés & 10 & $18,52 \%$ \\
\hline Francés- fongbe -inglés & 04 & $7,41 \%$ \\
\hline Inglés-francés-alemán & 02 & $3,70 \%$ \\
\hline 4. Textos cuatrilingües & 14 & $100 \%$ \\
\hline Chino-inglés-francés-español & 11 & $78,57 \%$ \\
\hline Francés-gungbe-inglés-fongbe & 03 & $21,43 \%$ \\
\hline 5. Textos quintilingües & 03 & $100 \%$ \\
\hline $\begin{array}{l}\text { Inglés-francés-español-chino- } \\
\text { portugués }\end{array}$ & 02 & $66,67 \%$ \\
\hline $\begin{array}{l}\text { Chino-inglés-francés-español- } \\
\text { árabe }\end{array}$ & 01 & $33,33 \%$ \\
\hline Suma & 3109 & \\
\hline
\end{tabular}

Tabla 1: Lenguas dominantes por la cantidad de textos

Cabe señalar que se observaron en algunos textos escritos errores gramaticales y ortográficos. A título de ejemplos, los siguientes: Lavage ouvert prope... (cf. 'propre' 'limpio'); Good príce (cf. 'price'); o palais de la boufe (cf. 'au palais de la bouffe' 'en el palacio de la comida'); la stars de la couture (cf. 'star' 'la estrella de la costura'); Multies services... (cf. 'multiservices' 
'multiservicios'). Por otro, en los textos chinos, los caracteres chinos están, a veces, juntos con el pinyin correspondiente, pero aparecía más el pinyin. En el paisaje lingüístico urbano analizado están presentes asimismo algunas palabras y sintagmas que dan cuenta del proceso de translenguar ${ }^{3}$ (cf. García 2009), mezclas de lenguas, etc.

\section{$5 \quad$ Interpretación de los resultados}

Aunque el corpus manejado todavía es limitado, hemos encontrado resultados interesantes que trataremos de explicar, con el fin de extraer algunas implicaciones didácticas surgidas de nuestro análisis.

\subsection{La presencia de las lenguas en el PL}

El PL de Cotonú y Abomey-Calavi recoge solo en parte la compleja realidad lingüística beninesa. Se observa una subrepresentación de las lenguas locales en comparación con la lengua francesa e incluso con otras lenguas extranjeras tales como el inglés, el chino y el español. Este PL funciona como imagen panorámica que refleja el contacto lingüístico en sus múltiples facetas (interferencias, fenómenos de interlengua, translenguar, etc.). El trabajo confirma que el PL es multilingüe casi por todas partes del mundo. Evidencia asimismo el dinamismo de las lenguas coexistentes en el país, la vitalidad etnolingüística, así como una fuente de información complementaria sobre el contexto sociolingüístico beninés o los datos procedentes de encuestas y censos lingüísticos. Se observa que las prácticas lingüísticas del país y los espacios urbanos se conforman un poco recíprocamente. Del mismo modo, el estudio ha facilitado datos sobre la identidad de algunos grupos lingüísticos locales e incluso extranjeros que vivifican la diversidad lingüística y cultural del país.

Este multilingüismo y las distintas lenguas del PL aparecen como un manantial de riquezas, una pasarela hacia la solidaridad y la comprensión recíproca. En dicha imagen, además de la lengua francesa, es de esperar que goce de un estatuto privilegiado el inglés, la lingua franca del mundo globalizado, y también que ocupen un lugar relevante otras lenguas extranjeras como la lengua castellana, el chino. Esta coexistencia de varias lenguas resulta de diferentes circunstancias tales como el imperialismo, el colonialismo (el caso de la lengua francesa), las migraciones, la globalización, el uso de lenguas de comunicación internacional (como el inglés, el español, el chino, el alemán), la identidad social y cultural, el anhelo por mantener y revitalizar las lenguas locales, los viajes de estudios al extranjero, así como la incidencia de la enseñanza-aprendizaje de las lenguas no maternas en el sistema educativo.

La lengua francesa domina en los textos tanto monolingües como multilingües del PL para indicar y reforzar la identidad francófona del país, su hegemonía, así como el estatus privilegiado del que dispone. Efectivamente, de acuerdo con el artículo primero de la Constitución de Benín de 1990 el francés es la (única) lengua oficial, mientras que coexiste con más de 73 lenguas nacionales o autóctonas (cf. Sossouvi 2017: 22). La lengua no solo se ve, sino también se siente (cf. Gade 2003: 446). Esto confirma y replantea el problema destacado

\footnotetext{
${ }^{3}$ El concepto de translenguar se refiere a prácticas lingüísticas inéditas, mixtas y compuestas de los hablantes bilingües y multilingües (cf. García 2009: 45, García/Wei 2014: 22); también es una estrategia pedagógica de los mismos (cf. Cenoz/Gorter 2017: 314).
} 
por Sossouvi $(2014,2017)$ acerca del repensamiento y la redefinición del estatus de la lengua francesa e incluso de las lenguas heredadas de la colonización en África. Este autor sugiere que se deje de considerar la lengua francesa (incluso otras lenguas heredadas de la colonización) como presencia extranjera o del yugo colonial en Benín o África, e invita a adoptarla como una lengua africana de más, ya que más de un siglo de cohabitación con las demás lenguas autóctonas le confiere la misma "nacionalidad" que ellas. Además, actualmente una persona puede tener dos o más primeras lenguas (para no decir lenguas maternas) y ningún país tiene el monopolio absoluto de una lengua. La hegemonía y visibilidad de esta lengua tienen relación con aspectos históricos, sociolingüísticos, económicos y políticos.

La aparición de las lenguas extranjeras en el PL va ligada al prestigio, al poder económico, a la demografía, a los procesos migratorios, al turismo, a los procesos de globalización económica, a los planes de estudio conducentes a títulos oficiales y a la negociación identitaria. La presencia del inglés se debe principalmente a su valor simbólico como lengua vehicular internacional que encarna la noción de modernidad, progreso, potencia económica y globalización. En otras palabras, refleja el proceso económico y cultural de la globalización o de la "McDonalización" de PL (cf. Heller 2003). Estimula el valor internacional, moderno, el éxito; da indicio de ciudades que están en vía de sofisticación, mostrando que ellas tampoco se han escapado del proceso de globalización. Este resultado coincide con los obtenidos en el PL de varias ciudades del mundo como Taipei (cf. Curtin 2009), Madrid (cf. Sáez Rivera/Castillo Lluch 2012). Por eso, Cormier (2015: 87) lo considera lengua mundial. La presencia de la lengua inglesa en el PL de las ciudades estudiadas confirma su desarrollo como lengua de comunicación internacional y transmite un valor connotativo (cf. Piller, 2003: 175). Resultados similares fueron encontrados por Ben-Rafael et al. (2006) quienes observaron la envergadura del inglés (entre el 25\% y el $75 \%$ de textos y rótulos) en el PL de Israel y lo consideraron una lengua "no extranjera". Por otra parte, su presencia también se debe a la buena relación que existe entre Benín y el gran vecino del este, Nigeria. A este respecto, la lengua del vecino perfila como una apertura al mercado laboral, la economía nigeriana y un instrumento conveniente en gran parte del continente africano. No obstante, esta preponderancia del inglés (en lugar de las lenguas nacionales) puede acarrear problemas de identidad, de poder e incluso de conminación (cf. Skutnabb-Kangas 2000, Phillipson 2003).

La combinación de lenguas más usual en el PL multilingüe estudiado era la de francés e inglés con otras lenguas, y puede achacarse al estatus de ambas lenguas en el país, así como a la globalización del inglés. Este tiene el estatuto de lengua extranjera privilegiada, obligatoria en el país desde la educación infantil hasta la enseñanza superior. Por ello Sossouvi (2017: 24) cree que la lengua shakespeariana está convirtiéndose implícitamente en la segunda lengua oficial de Benín. Este hallazgo está en línea con las conclusiones de Backhaus $(2006,2007)$ acerca de la influencia del inglés en el PL de Tokio (hasta el 98\%). La supremacía del francés en el PL potencia y refuerza el sentimiento de pertenencia a la comunidad francófona. Esta situación también puede deberse al hecho de que el dominio del francés y del inglés sea condición innegociable para acceder a algunas ofertas de trabajo, ascender profesionalmente e incluso para cursar estudios de posgrado y doctorado en el país.

La visibilidad de elementos bilingües o multilingües indica que la población beninesa valoriza la diversidad lingüística y cultural. Además, algunos benineses promueven la utilización de 
varias lenguas en sus carteles, a veces sin entenderlas; solo quieren demostrar su amor por estas lenguas, mostrar un exotismo que responde a la alteridad surgida en la confluencia de varias culturas, reconocer el valor de estos carteles que muestran la riqueza y la conveniencia de la diversidad lingüística en estas urbes.

En las calles estudiadas también existen otras lenguas extranjeras como el español, el chino y el alemán. Su presencia parece bien dirigida a los migrantes extranjeros locutores de estas lenguas, a turistas, o en algunos casos podría servir para reforzar la idea de ciudades cosmopolitas y modernas. Además, constituye una buena señal del apego de los benineses, de algunos exalumnos de estas lenguas e incluso de los que cursaron estudios de posgrado o doctorado en los países de las distintas lenguas extranjeras. Las intenciones de los emisores del PL pueden ser crear espacios culturales y lingüísticos de la oralidad (cf. Calvi 2016), instaurar un punto de contacto que posibilita la integración. Así, el PL nos puede ofrecer informaciones significativas sobre las actividades de las comunidades inmigrantes de estas lenguas.

Apenas se observan las lenguas de grupos inmigrantes africanos; y una posible explicación para esto es que, el conjunto de las lenguas africanas falta de transcripción escrita y todavía no han alcanzado gran difusión, ni tienen alcance internacional como las lenguas de las principales potencias económicas. Esta realidad insinúa que hoy en día, una gran parte de los emisores de esta modalidad escrita, por un sentido práctico, prefiere utilizar una lengua con proyección y prestigio internacional.

Los elementos monolingües en chino registrados demuestran que los chinos que viven en el país procuran mantener y exhibir su propia cultura, y al mismo tiempo intentan incorporarse en la sociedad local beninesa. Pueden explicarse en parte por el hecho de que los chinos de ultramar se empeñan en utilizar su L1 en cualquier país extranjero. Además, cuando se dedican a actividades comerciales usan su L1 para comunicarse, con el fin de mantener el negocio entre inmigrantes chinos y fidelizar a la clientela china. El uso de pinyin supone un exceso o sobreinformación para la comunidad sinohablante; por contra, puede ser por ayudar a la comunidad local a saber algo de chino o una estrategia de promoción internacional de la lengua china. La presencia de caracteres chinos en el espacio público beninés es un buen indicador de los cambios en la economía mundial. Resultados similares han sido obtenidos por Huebner (2006) en los chinos de las calles Yawarat y Charoen Krung de Bangkok.

Las observaciones del PL insinúan que el público meta y los consumidores hablan varias lenguas. Los textos bilingües o multilingües están presentes para acoger y atraer a los clientes locutores de estas lenguas. Eso indica que las lenguas pueden coexistir o que los autores de los textos escritos están dispuestos a aceptar y facilitar la utilización de todas las lenguas. La identidad colectiva de ambas ciudades es, pues, una identidad multilingüe consolidada.

A pesar de la fuerte presencia del bilingüismo o multilingüismo en el paisaje lingüístico de Cotonú y Abomey-Calavi, todavía no existen conflictos o relaciones tensas entre los grupos lingüísticos, como demuestra el hecho de que no hay mensajes públicos vandalizados. Por tanto, los distintos grupos lingüísticos o étnicos conviven en paz, armonía y unidad. Asimismo, el trabajo revela que los inmigrantes que hablan estas lenguas contribuyen al "diseño" o al "sentido" de las urbes como lo señalan los estudios de Stroud (2016: 1) 
El valor de la existencia también se puede ver en el paisaje lingüístico de ambas ciudades. La presencia de algunas lenguas autóctonas (fongbe, yoruba, gungbe, etc.) en el PL es una fuente de comodidad para sus locutores que, a veces, temen la desaparición o la insignificancia de sus lenguas en el país. Dado que estas lenguas son visibles en las calles, supone un respaldo importante y significa que los grupos lingüísticos esperan que sus lenguas sean oficiales o puedan ser una realidad en tiempos venideros. Cabe mencionar que muchas lenguas nacionales han sido invisibilizadas del espacio público, por eso, es recomendable asimismo crear más señalética en lenguas locales.

\subsection{Algunas implicaciones didácticas}

Las desviaciones lingüísticas registradas en el PL nos ofrecen un panorama de la situación actual de estas lenguas usadas en el país y, más especialmente, de las situaciones educativas de sus autores. Nos indican algunas carencias en el conocimiento de estas lenguas. Además, reflejan la cultura lingüística del país donde la alternancia o mezcla de lenguas y los translenguares son muy frecuentes en el contexto informal, con el fin de optimizar la comunicación. Se puede detectar y analizar estos errores para ayudar a conocer el estado de aprendizaje de las lenguas concernidas y pueden servir como base para los estudios sobre los hablantes o aprendientes multilingües. Parecidos resultados han sido encontrados por Ma (2017) al identificar errores en el PL de Valencia.

Las lenguas extranjeras que forman parte de los textos escritos en las vías públicas pueden ser una fuente adicional de input y, además, la mayor o menor presencia de una lengua meta en el PL puede influir en la valoración de las lenguas adicionales del discente, así como su motivación hacia su aprendizaje. Los resultados de este trabajo sugieren que el PL constituye una herramienta heurística que puede ayudar a los discentes a desempeñar un papel de actores informados sobre los asuntos y desafíos relacionados con el plurilingüismo (cf. Dagenais et al. 2009).

El PL presenta varias bazas para el proceso de enseñanza-aprendizaje y puede ser didactizado. Expone al aprendiente a la lengua meta y le permite entrar en contacto con un nuevo género de texto gratuitamente y un corpus de textos auténticos en la lengua meta. También le impulsa tanto al aprendizaje autónomo como informal, y puede contribuir al desarrollo de su competencia intercultural. El discente puede aprovechar al máximo los contenidos gramaticales, léxicos, socioculturales y pragmáticos. Se puede utilizar el PL como apoyo a las actividades docentes, para proponer actividades.

La experiencia diaria de los aprendientes y los objetivos curriculares del docente puede tener como punto de referencia el PL. Se puede utilizar este último para crear o realizar actividades escolares, unidad didáctica. Por ejemplo, se pedirá al alumnado que investigue sobre el PL de su propio espacio vivencial, con el fin de desarrollar una pedagogía de alfabetización multimodal para que comprenda y reconozca los distintos significados de esta modalidad escrita, con el propósito de ubicarla e interpretarla desde un punto de vista crítico. Este contacto de los educandos con el PL, pues con las diversas lenguas y culturas de su entorno, participa efectivamente en la contextualización de las actividades de clase, permite hacer clases participativas y entretenidas, así como reforzar las competencias en las diferentes lenguas implicadas como parte de una educación plurilingüe y multilingüe. 
Concretamente, una unidad o sesión didáctica sobre el PL puede consistir en pedir a los estudiantes que hagan lectura de su entorno, de la ciudad. Los aprendientes pueden recorrer su barrio, los espacios públicos, en busca de escritos en diferentes lenguas, o en nuestro caso, en lengua castellana. Van a sacar fotos de todos los elementos del PL. Luego, los estudiantes van a producir textos de acuerdo con su interés y nivel, por ejemplo: clasificar las fotos y desarrollar un argumento para justificarlas y explicar la(s) causa(s), etc. Finalmente, pueden producir el soporte visual necesario (póster, vídeo, etc.) para presentar su comprensión del PL y su entorno social (historia migratoria y situación de las lenguas, etc.), en nuestro caso, los discentes redactan su discurso o producen textos en español, e igualmente pueden intentar corregir los errores detectados en esta modalidad escrita. También pueden buscar ejemplos de contenidos gramaticales o léxicos estudiados en clase en estas muestras lingüísticas de la ciudad.

De hecho, las fotos del PL sacadas por los discentes constituyen un material didáctico auténtico a partir del cual pueden construir y desarrollar sus saberes lingüísticos, sociales e interculturales. Se puede comentar las fotos sacadas, liberando así la expresión en los aprendientes, todo el potencial que poseen, permitiéndoles explorar en profundidad el paisaje lingüístico investigado. Los aprendientes pueden diseñar carteles y pósteres dirigidos a otros estudiantes residentes en otra región. Estos desplazamientos urbanos efectuados los ponen en contacto con una variedad de textos escritos. Los discentes se vuelven los principales autores del proceso de aprendizaje. Por añadidura, es una práctica pedagógica de tipo experimental, ya que los discentes observan y experimentan el entorno que los rodea. Por consiguiente, se puede utilizar la vida diaria de los aprendientes, el paisaje lingüístico como experiencia y estrategia de enseñanza-aprendizaje, e incluso para moldear personas participativas, creativas y críticas. El docente puede utilizar el PL como un medio de adquisición de competencias y conocimientos, como una herramienta de evaluación, y como refuerzo a una tarea determinada o en actividades adicionales dependiendo del estudiantado.

\section{$6 \quad$ Reflexiones finales}

Este trabajo fue diseñado para sacar a luz el paisaje lingüístico de dos ciudades beninesas, analizar la presencia de las distintas lenguas en su espacio público y ver cómo se puede utilizar como recurso didáctico en el aprendizaje de lenguas extranjeras. El estudio del PL evidencia el multilingüismo, la multiculturalidad y el cosmopolitismo que caracterizan las ciudades de Cotonú y Abomey-Calavi, así como la distribución asimétrica de las lenguas que tienen presencia en los espacios públicos. Proporciona materiales lingüísticos apreciados para la enseñanza-aprendizaje de lenguas extranjeras en el país, donde las lenguas y las sociedades se fusionan y se mezclan. Como es lógico, en un contexto francófono de África, el PL revela el peso y el prestigio de la lengua predominante, el francés. Pero el trabajo atestigua asimismo la presencia de lenguas extranjeras como el inglés, el español, el chino y algunas lenguas autóctonas, como el fongbe, el yoruba, el gungbe, en el tejido público urbano de las dos ciudades beninesas. Desde una perspectiva lingüística, se puede afirmar que la cantidad de textos analizados da prueba de la vitalidad de las lenguas de los textos, sus valores políticos, económicos y culturales, en otras palabras, el impacto de la política lingüística del país. Estos hallazgos sugieren que, en general, el PL de estas ciudades es un poco comparable al de grandes ciudades como París, Londres, Madrid, Nueva York, Tokio, Kaohsiung, Taipéi, Hong Kong, 
Shanghái, etc. Sin embargo, debemos reconocer que la heterogeneidad lingüística de ambas urbes es superior a la experimentada en su paisaje lingüístico. Los textos escritos del PL no tienen en cuenta las prácticas multilingües locales en toda su complejidad. En otros términos, a este multilingüismo del PL, hay que añadir el multilingüismo dinámico mucho más complejo de la población beninesa.

Los resultados de esta investigación apoyan la idea de que el estudio del paisaje lingüístico constituye una aportación considerable al estudio del plurilingüismo (multilingüismo) en el mundo (cf. Backhaus 2006, 2007), a la planificación y las políticas lingüísticas; puede constituir un valioso recurso didáctico en el aula de lengua extranjera, un medio de comunicación, e incluso ayuda a redefinir, a reconsiderar la noción clásica de contacto lingüístico. Además, permite una enseñanza o un aprendizaje orientado a la acción, y puede ser fuente de numerosos tipos de actividades. Por último, este trabajo invita a visibilizar más las lenguas nacionales, hacer para que sean más representativas en los espacios públicos.

\section{Referencias bibliográficas}

Backhaus, Peter (2006): "Multilingualism in Tokyo: A look into the linguistic landscape". In: Gorter, Durk (ed.): Linguistic Landscape: A New Approach to Multilingualism. Clevedon/Buffalo/Toronto, Multilingual Matters: 52-66.

Backhaus, Peter (2007): Linguistic landscapes: A comparative study of urban multilingualism in Tokyo. Clevedon/Buffalo/Toronto: Multilingual Matters.

Barni, Monica/Bagna, Carla (2015): "The critical turn in LL: New methodologies and new items in LL". Linguistic Landscape 1/1/2: 6-18.

Ben-Rafael, Eliezer et al. (2006): "Linguistic landscape as symbolic construction of the public space: The case of Israel”. International Journal of Multilingualism 3/1: 7-30.

Blackwood, Robert (2015): "LL explorations and methodological challenges: Analysing France's regional languages". Linguistic Landscape 1/1/2: 38-53.

Boschung, Susanne (2016): "Le paysage linguistique: reflet d'une réalité bilingue à Moncton, Nouveau-Brunswick, Canada”. Travaux neuchâtelois de linguistique 64: 161-180.

Bourhis, Richard Y./Landry, Rodrigue (2002): "La loi 101 et l'aménagement du paysage linguistique au Québec". In: Bouchard, Pierre/Bourhis, Richard Y. (eds.): L'aménagement Linguistique au Québec: 25 Ans D'application de la Charte de la Langue Française. Québec, Publications du Québec: 107-132.

Calvi, Maria Vittoria (2016): "La oralidad en el paisaje lingüístico latinoamericano en Milán". In: Bañón Hernández, Antonio Miguel et al. (eds.): Oralidad y análisis del discurso. Homenaje a Luis Cortés Rodríguez. Almería, Editorial Universidad de Almería: 123-139.

Cenoz, Jasone/Gorter, Durk (2006): "Linguistic landscape and minority languages". International Journal of Multilingualism 3/1: 67-80.

Cenoz, Jasone/Gorter, Durk (2008): "The linguistic landscape as an additional source of input in second language acquisition". International Review of Applied Linguistics in Language Teaching (IRAL) 46/3: 267-287. doi.org/10.1515/IRAL.2008.012.

Cenoz, Jasone/Gorter, Durk (2017): “Translanguaging as a pedagogical tool in multilingual education". In: Cenoz, Jasone/Gorter, Durk/May, Stephen (eds.): Language Awareness and 
Multilingualism. Encyclopedia of Language and Education. Vol 6. $3^{\text {rd }}$ ed. Cham/New York, Springer: 309-321.

Cormier, Gail (2015): “Le paysage linguistique en milieu minoritaire: une étude de l'affichage commercial à Saint-Boniface, au Manitoba”. Minorités linguistiques et société 5: 84-99. doi.org/10.7202/1029108ar.

Curtin, Melissa L. (2009): "Languages on display: Indexical signs, identities and the linguistic landscape of Taipei”. In: Shohamy, Elana/Gorter, Durk (eds.): Linguistic Landscape: Expanding the Scenery. New York/London, Routledge: 221-237.

Dagenais, Diane et al. (2009): "Linguistic landscape and language awareness". In: Shohamy, Elana/Gorter, Durk (eds.): Linguistic Landscape: Expanding the Scenery. New York/London, Routledge: 253-269.

Gade, Daniel W. (2003): "Language, identity, and the scriptorial landscape in Quebec and Catalonia". The Geographical Review 93/4: 429-448.

García, Ofelia (2009): Bilingual education in the 21st century: A global perspective. Malden, MA: Wiley-Blackwell.

García, Ofelia/Wei, Li (2014): Translanguaging: Language, bilingualism and education. New York: Palgrave Macmillan.

Hancock, Andy (2012): "Capturing the linguistic landscape of Edinburgh: A pedagogical tool to investigate student teachers' understandings of cultural and linguistic diversity". In: Hélot, Christine et al. (eds.): Linguistic Landscapes, Multilingualism and Social Change. Frankfurt am Main etc., Lang: 249-266.

Heller, Monica (2003): "Globalization, the new economy, and the commodification of language and identity". Journal of Sociolinguistics 7/4: 473-492. doi.org/10.1111/j.14679841.2003.00238.x.

Huebner, Thom (2006): “Bangkok's linguistic landscapes: Environmental print, codemixing and language change". International Journal of Multilingualism 3/1: 31-51.

INSAE (2015): RGPH4: que retenir des effectifs de population en 2013?. Cotonou: Direction des études démographiques.

Landry, Rodrigue/Bourhis, Richard Y. (1997): "Linguistic landscape and ethnolinguistic vitality: An empirical study". Journal of Language and Social Psychology 16/1: 23-49.

Ma, Yujing (2017): "El paisaje lingüístico chino-español de la ciudad de Valencia: una aproximación a su estudio". Lengua y migración / Language and Migration 9/1: 63-84.

Malinowski, David (2015): "Opening spaces of learning in the linguistic landscape". Linguistic Landscape 1/1/2: 95-113.

Masai, Yasuo (1972): Living Map of Tokyo. Tokyo: Jiji Tsushinsha.

Phillipson, Robert (2003): English-only Europe? Challenging language policy. London/New York: Routledge.

Piller, Ingrid (2003): “Advertising as a site of language contact". Annual Review of Applied Linguistics 23: 170-183.

Pons Rodríguez, Lola (2012): El paisaje lingüístico de Sevilla. Lenguas y variedades en el escenario urbano hispalense. Sevilla: Diputación de Sevilla.

Prego Vázquez, Gabriela/Zas Varela, Luz (2018): "Paisaje lingüístico. Un recurso TIC-TACTEP para el aula”. Lingue e Linguaggi 25: 277-295. doi: 10.1285/i22390359v25p277. 
Pujol Berché, Mercè/Rámila Díaz, Noemí (2018): "Estereotipos sobre España en el paisaje lingüístico de París”. Amnis. doi: 10.4000/amnis.3457.

Regueira, Xosé Luís/López Docampo, Miguel/Wellings, Matthew (2013): "El paisaje lingüístico en Galicia". Revista internacional de lingüistica iberoamericana (RILI) 21: $39-62$.

Rosenbaum, Yehudit et al. (1977): "English on Keren Kayemet street". In: Fishman, Joshua A./Cooper, Robert L./Conrad, Andrew W. (eds.): The Spread of English: The Sociology of English as an Additional Language. Rowley MA, Newbury House: 179-196.

Rowland, Luke (2013): "The pedagogical benefits of a linguistic landscape project in Japan". International Journal of Bilingual Education and Bilingualism 16/4: 494-505.

Sabatier, Cécile/Moore, Danièle/Dagenais, Diane (2013): "Espaces urbains, compétences littératiées multimodales, identités citoyennes en immersion française au Canada". Glottopol. Revue de sociolinguistique en ligne 21: 138-161. http://glottopol.univ-rouen.fr/telecharger/numero_21/gpl21_09sabatier_moore_dagenais.pdf [16.01.2018].

Sáez Rivera, Daniel M./Castillo Lluch, Mónica (2012): "The human and linguistic landscape of Madrid (Spain)". In: Hélot, Christine et al. (eds.): Linguistic Landscapes, Multilingualism and Social Change. Frankfurt am Main etc., Lang: 309-328.

Skutnabb-Kangas, Tove (2000): Linguistic genocide in education - Or worldwide diversity and human rights?. Mahwah, NJ: Lawrence Erlbaum Associates.

Sossouvi, Laurent-Fidèle (2014): "La lengua castellana en Benín: tendencias actuales después de seis décadas". In: Serrano Avilés, Javier (ed.): La enseñanza del español en África subsahariana. Madrid, Editorial Catarata: 100-126.

Sossouvi, Laurent-Fidèle (2016): "Enseñanza y aprendizaje del ELE en Benín: ¿qué puede aportar la adaptación del MCER? Teaching and learning of SFL in Benin: What can provide the adaptation of the CEFR?". Tejuelo. Didáctica de la Lengua y la Literatura. Educación 23/1: 36-62. doi: 10.17398/1988-8430.23.1.36.

Sossouvi, Laurent-Fidèle (2017): Replantear la enseñanza-aprendizaje de ELE en Benín: los posibles aportes de la adaptación del MCER. Balti: Editorial Académica Española.

Spolsky, Bernard/Cooper, Robert L. (1991): The Languages of Jerusalem. Oxford: Clarendon Press.

Stroud, Christopher (2016): "Turbulent linguistic landscapes and the semiotics of citizenship". In: Blackwood, Robert/Lanza, Elizabeth/Woldemariam, Hirut (eds.): Negotiating and Contesting Identities in Linguistic Landscapes. London etc., Bloomsbury Academic: 3-18.

Tulp, Stella (1978): "Reklame en tweetaligheid. Een onderzoek naar de geografische verspreiding van franstalige en nederlandstalige affiches in Brussel". Taal en sociale integratie 1: 261-288.

Zas Varela, Luz/Prego Vázquez, Gabriela (2018): “A view of linguistic landscapes for an ethical and critical education”. In: DePalma, Renée/Pérez-Caramés, Antía (eds.), Galician Migrations: A Case Study of Emerging Super-diversity. Cham, Springer: 249-264. 
Apéndices: algunos textos del paisaje lingüístico

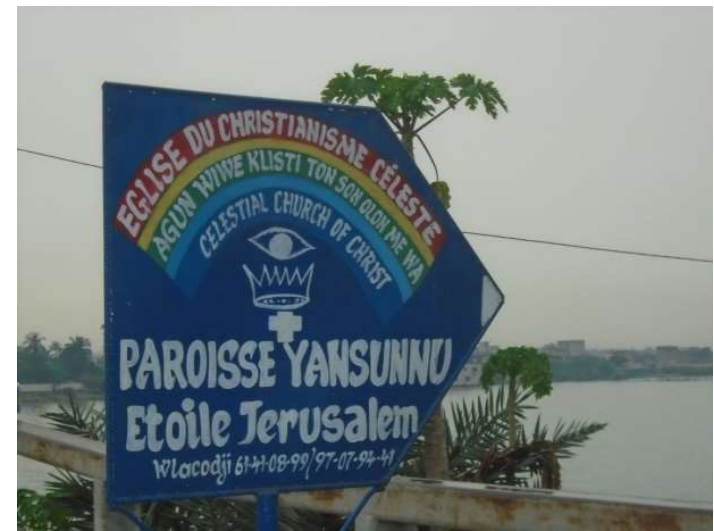

Foto 1. Francés-gungbe-inglés-fongbe

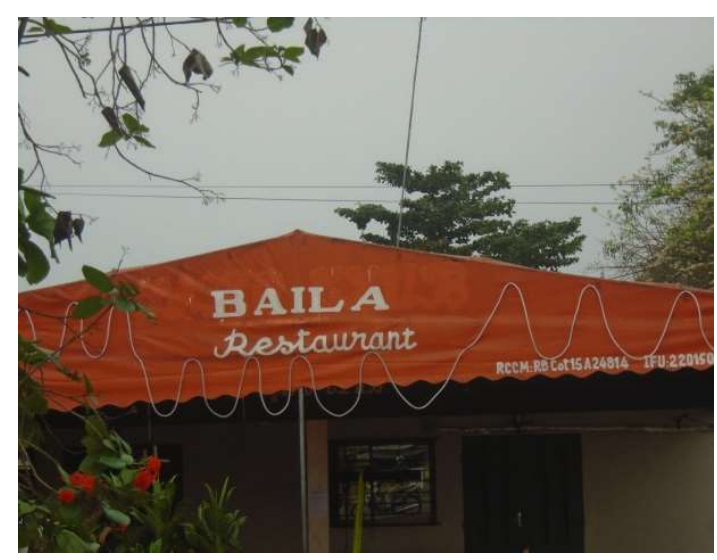

Foto 3. Español-francés

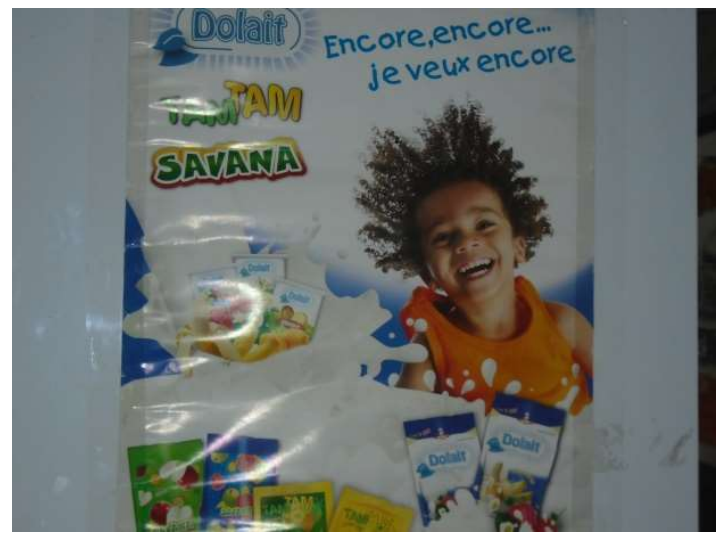

Foto 5. Español-francés

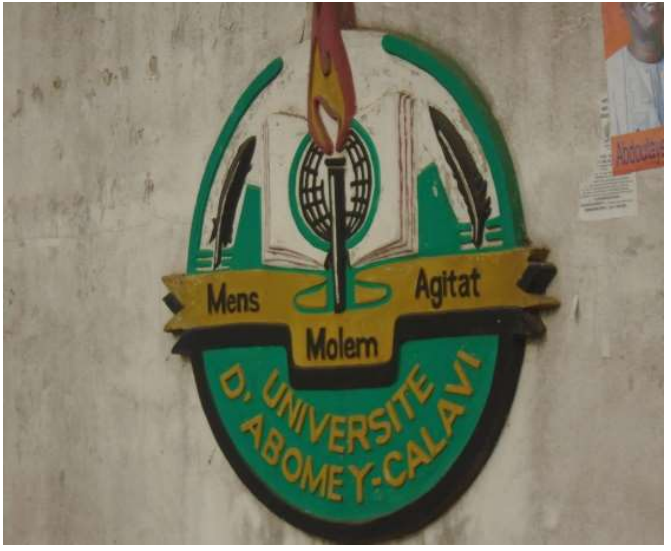

Foto 2. Latín-francés

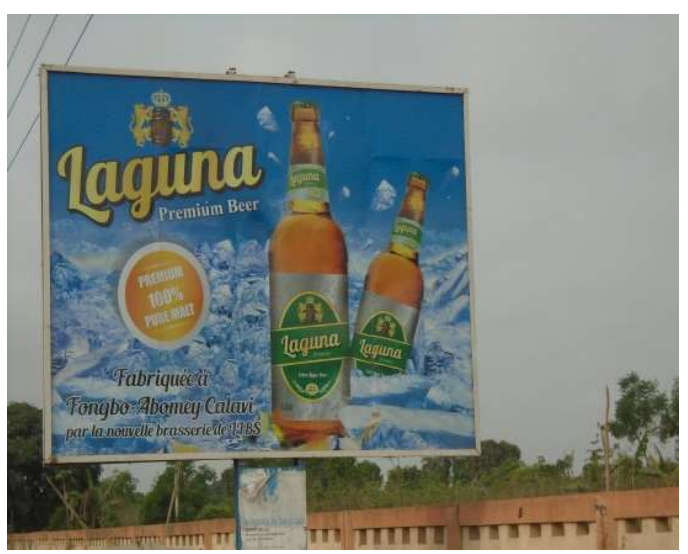

Foto 4. Francés-español-inglés

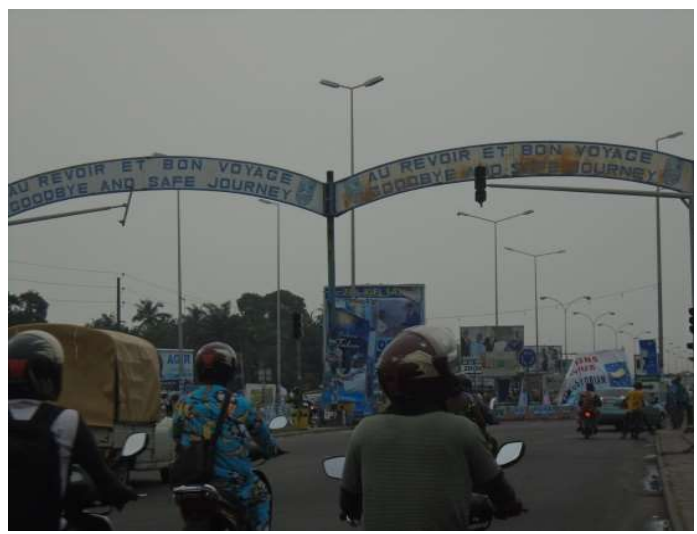

Foto 6. Francés-inglés 


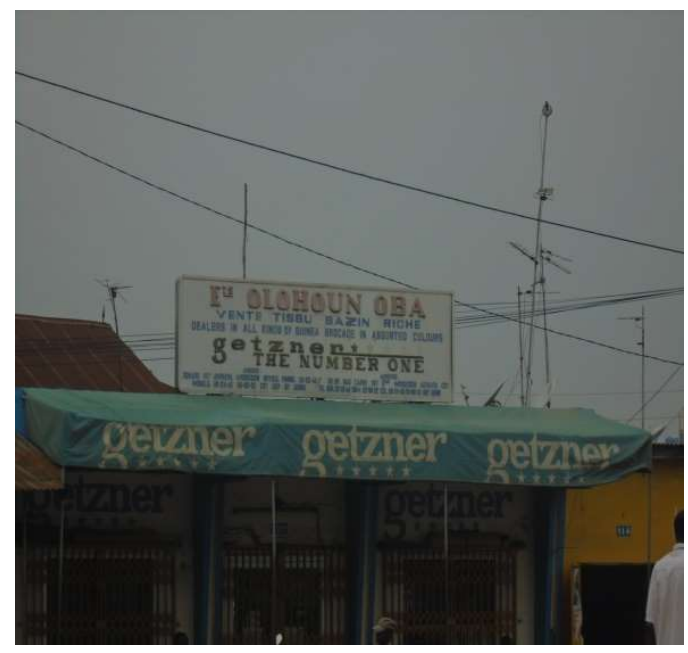

Foto 7. Inglés-francés-yoruba

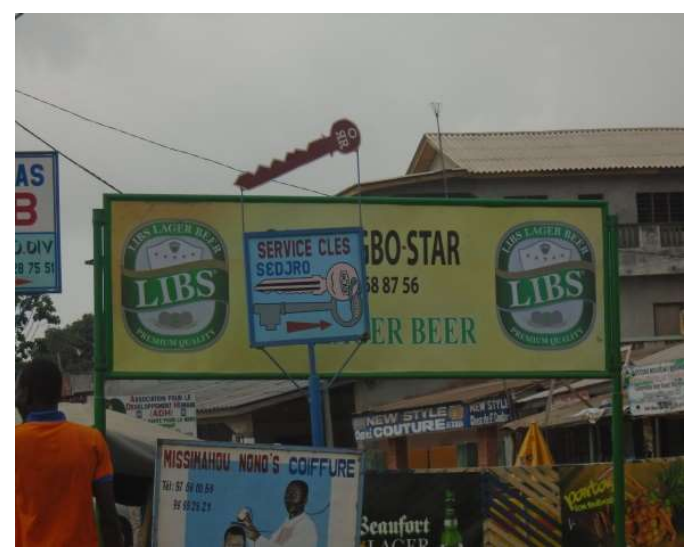

Foto 9. Francés-fongbe

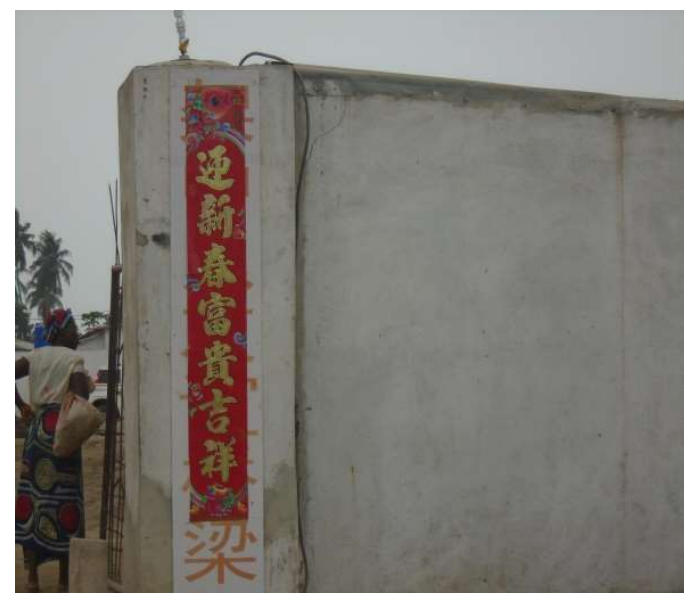

Foto 11. Chino

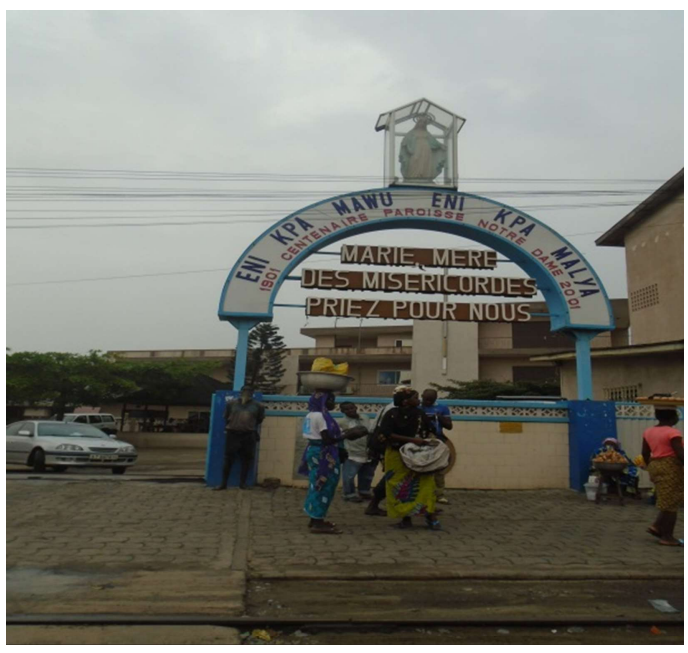

Foto 8. Francés-fongbe

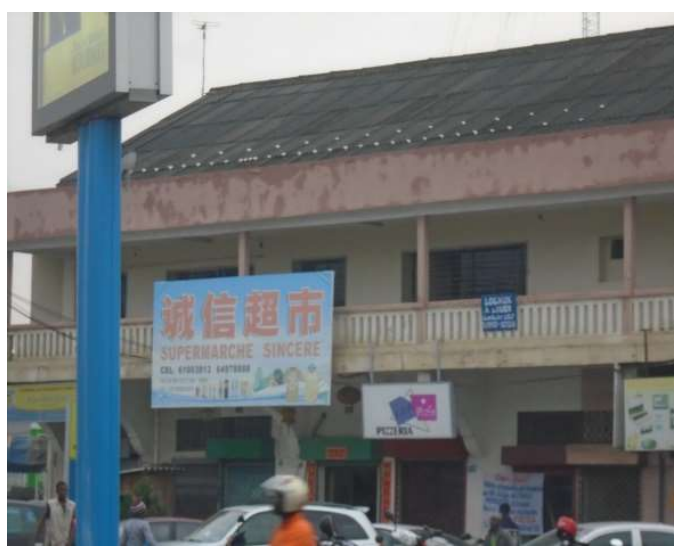

Foto 10. Chino-francés

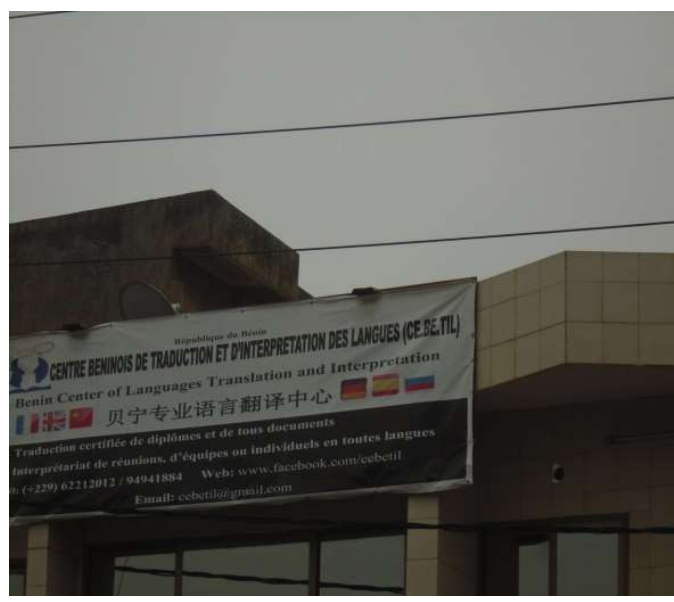

Foto 12. Chino-inglés-francés 